\title{
Contenido generado por el usuario: aproximación al estado de la cuestión
}

\author{
Por Elvira García-De-Torres
}

\begin{abstract}
Resumen: Se analizan las relaciones conceptuales entre lo que se ha denominado periodismo ciudadano, periodismo participativo y periodismo de crowdsourcing o fuentes masivas en el contexto de la evolución del contenido generado por el usuario (CGU) en los medios informativos. Seguidamente se hace una revisión de la investigación sobre la participación de los usuarios en los medios informativos, desde los primeros estudios de interactividad hasta las líneas actuales, que abordan temas concretos como la calidad o el control.
\end{abstract}

Palabras clave: Contenido generado por el usuario, UGC, Periodismo digital, Periodismo ciudadano, Investigación, Periodismo participativo, Web 2.0.

\section{Title: User generated content: a state of the situation}

Abstract: The conceptual relationships between what has been called citizen journalism, participatory journalism and crowdsourcing journalism in the context of the evolution of user-generated content (UGC) in the media are examined. The research on user participation in the media, since the first studies of interactivity to the most recent work addressing specific issues such as quality or control, is also reviewed.

Keywords: User generated content, UGC, Citizen journalism, Online journalism, Research, Participatory journalism, Web 2.0.

García-De-Torres, Elvira. "Contenido generado por el usuario: aproximación al estado de la cuestión”. El profesional de la información, 2010, noviembre-diciembre, v. 19, n. 6, pp. 585-594.

DOI: 10.3145/epi.2010.nov.04

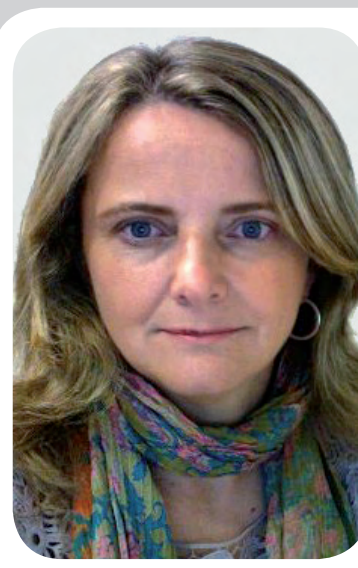

Elvira García-De-Torres es doctora en ciencias de la información por la Universidad Complutense de Madrid y máster en nuevas tecnologías de la comunicación y de la información. Desde 2005 dirige el Observatorio de Investigación en Medios Digitales del Instituto de Diseño, Innovación y Tecnología. Imparte docencia de periodismo digital en la Universidad CEU-Cardenal Herrera, en el Máster de Comunicación Digital CEU-UCH y el Máster en Nuevo Periodismo de la Universitat Internacional Valenciana. Desde 2002 ha dirigido ocho proyectos de investigación sobre medios digitales con financiación $I+D$ del Ministerio de Ciencia e Innovación -UCH y ha realizado estancias de investigación en la Universidad de Minnesota y la Universidad de Texas. Es vicepresidenta de la Associació de Mitjans Digitals de la Comunitat Valenciana y colabora en el diario Las Provincias.

\section{Introducción}

La publicación de contenido generado por los usuarios (CGU) en el ámbito de la información periodística no es una novedad; basta recordar las Cartas al director. Sin embargo, sí lo es el incremento de espacios para las contribuciones de los usuarios en los medios informativos y la creciente integración de estos contenidos en los espacios, hasta ahora, reservados a los profesionales de la información.

En We media, Shayne Bowman y Chris Willis (2002) relacionan el contenido generado por el usuario en el periodismo con una de las formas de periodismo participativo, junto con los foros, los weblogs y la escritura colaborativa. Más recientemente, Alfred Hermida y Neil Thurman (2008) lo definen como el proceso en el que cualquier persona tiene la posibilidad de hacer contribuciones a las publicaciones editadas profesionalmente.

Artículo recibido el 14-09-10

Aceptación definitiva: 06-10-10 


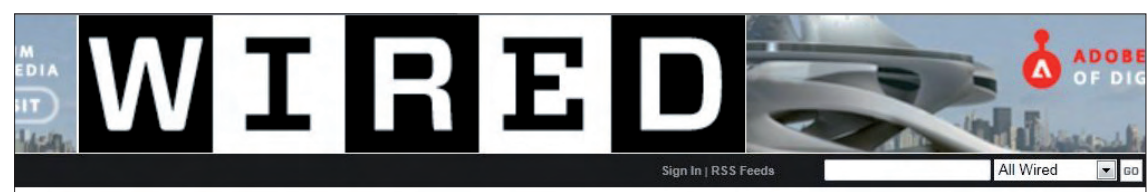

/ MAGAZINE

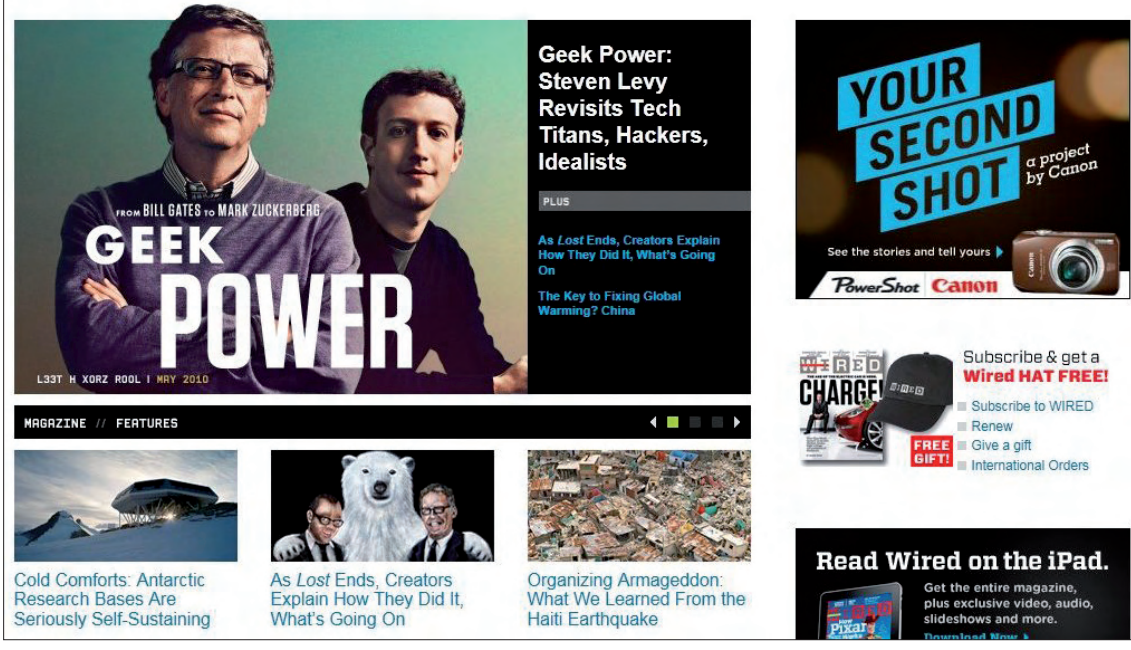

Figura 1. La palabra crowdsourcing (crowd + outsourcing) o participación de la gente en actividades (resolución conjunta de problemas, colaboraciones en medios de comunicación, etc.), la usó por primera vez Jeff Howe en la revista de divulgación científica Wired en 2006

los "libros de guerra", por ser un contenido más creativo, constituyen el puente entre el periodismo tradicional de interactividad selectiva y el periodismo de interactividad comunicativa moderno.

Con internet se abren nuevos cauces para el CGA. Cuando Sheizaf Rafaeli, en diálogo con John Newhagen (Newhagen y Rafaeli, 1996) apunta la apelación sensorial multimedia, la ruptura de la linealidad, la desaparición de rutas prefijadas en el tráfico de información, la elasticidad de la sincronía y la interactividad como características de la Red, Newhagen responde que la arquitectura distribuida y la digitalización del mensaje son las características menos visibles para un usuario, pero son las que marcan la diferencia frente a anteriores tecnologías; sostiene, con Mark R.

para futuros temas y reportajes, y la función vigilante de la audiencia. Todas ellas requieren un cierto esfuerzo; jerarquizar las noticias posiblemente se encuentre en la base de la pirámide. Las escasas prácticas de crowdsourcing o periodismo de fuentes masivas a través de blogs realizadas hasta ahora han puesto de manifiesto el valor añadido de los usuarios en la cobertura de hechos especializados o de carácter social (Muthukumaraswamy, 2010; Bradshaw, 2007).

\section{"Puede considerarse CGU toda aportación pública del usuario que no constituya una copia, ni un modo de vida (profesionalización)"}

\section{CGU en los medios informativos}

Antecedentes de lo que denominamos contenido generado por el usuario son el contenido generado por el lector (CGL) o el radioyente (CGR) o, en general, el contenido generado por la audiencia (CGA) antes de la llegada de la informática a las comunicaciones, es decir, las intervenciones telefónicas en las emisoras, publicaciones alternativas, cartas al director e incluso, como apunta Stefanie Markovits (2008), cartas publicadas en los diarios durante la cobertura de una guerra. Las cartas personales publicadas en gacetas y diarios, incluso los informes de los generales que engrosaban
Levy, que en la arquitectura distribuida reside el verdadero poder de internet y que de ella se deriva la interactividad (Newhagen y Levy, 1996).

\section{"La capacidad de usar o manipular el contenido en el medio de recepción ha sido hasta ahora muy limitada"}

La noción de CGU es extraña al paradigma clásico, no sólo porque la capacidad de usar o manipular el contenido en el medio de recepción ha sido hasta ahora muy limitada sino porque el flujo informativo de retorno tiene valor, fundamentalmente, en terminología de Gerhard Maletzke (1963), como fuente de compulsión o presión. En el periodismo, la informática y el desarrollo de internet abren paso al usuario activo productor de contenidos.

En los primeros años, el CGU está presente en los grupos de noticias, foros y tablones de anuncios, las páginas web editadas por usuarios y las zonas de charla; se trata de formatos con bastantes limitaciones.

La disponibilidad de las plataformas gratuitas de fácil edición para la publicación de blogs amplía notablemente los cauces del CGU y se hace más visible el poder de transformación de la estructura, en la que se abre paso la denominada web 2.0 (Blood, 2002; García-De-Torres, 2004; Rojas et al., 2005). No en vano 


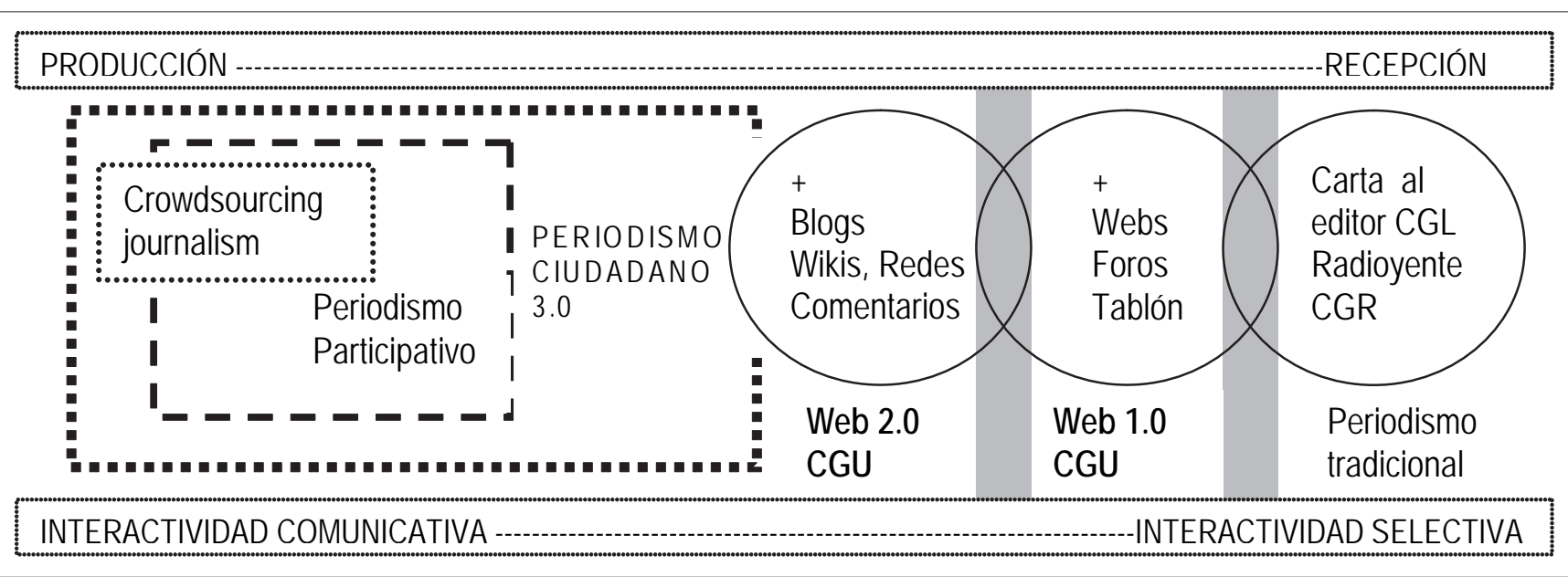

Figura 2. Evolución del contenido generado por la audiencia (CGA)

la interactividad es una característica constitutiva del lenguaje de la web (Rost, 2010). Wu (2010) considera que la web 2.0 supone un cambio de hábito participativo.

En este contexto, Dan Gillmor (2004) acuña el término "periodismo ciudadano" y elabora un argumentario que pone a la defensiva a sectores académicos y profesionales: Es un hecho que miembros individuales de la sociedad pueden desempeñar algunas de las funciones institucionalizadas de medios informativos y agencias (Domingo et al., 2008), pero Alejandro Rost (2010) se pregunta si pueden las contribuciones de los ciudadanos llamarse «periodismo» y qué contribuciones cabrían en este término.

Al pensar en la cuestión en términos de lo qué constituye "información periodística" resulta más claro cómo enfocar este problema de conceptos. Así, cuando un ciudadano da a conocer un hecho enriquece la producción informativa y amplía la red de captación -uno de las grandes retos de las organizaciones informativas desde el siglo XIX-, alimentando el flujo de noticias. Ahora bien, diversos estudios han puesto de manifiesto su debilidad por:

a) La dependencia respecto de la agenda de los medios informativos (García y Capón, 2004), confirmada por el responsable de Global voices en el X Symposium on online journalism, en 2010, al considerar que la influencia de los ciudadanos en la agenda es mínima.

b) Periodicidad insuficiente, como ponen de manifiesto Stephen Lacy et al. (2010) tras examinar 53 medios y 86 blogs ciudadanos: sólo el 27,7\% actualiza diariamente el sitio, por lo que complementan pero difícilmente podrían sustituir a los medios tradicionales.

c) Limitaciones en los métodos de producción de noticias, como sugiere un informe del Pew Research Center realizado en 2009, según el cual los sitios de periodismo ciudadano citan una sola fuente en porcentaje mayor que los sitios de periodismo tradicional. Reich (2008) ha estudiado la producción de información ciudadana en el sitio ciudadano Scoop y concluye que los editores añaden fuentes informativas porque los ciudadanos no saben que aquellos afectados negativamente por los contenidos, deberían poder dar su punto de vista. Según este mismo estudio, los periodistas ciudadanos están bien equipados para informar cuando:

- los hechos no dependen de una fuente habitual;

- pueden obtener la información de fuentes técnicas o textuales así como de conocidos;

- se trata de temas que no requieren negociar o interrogar fuentes, confrontar versiones o un procesamiento elaborado de la información;

- se trata de eventos que pueden ser observados directamente.

El cierre de la edición internacional de OhMyNews en agosto de 2010, debido a la dificultad para controlar la calidad de los contenidos, añade un elemento más al debate. Se trata, por otra parte, en cuanto al control, de un problema común a los medios tradicionales.

También en los viejos diarios convencionales el periodismo ciudadano o participativo avanza posiciones (Paulussen y Ugille, 2008).

La primera señal de apuesta por la web 2.0 en los medios tradicionales fue creación de blogs de autor a principios de la década del 2000. A partir de 2006, con la implementación de canales o zonas para la publicación de noticias ciudadanas en medios de alcance como I report (CNN) o You witness news (Reuters), el periodismo participativo se consolida.

El grado de aceptación y desarrollo varía. Al margen de medios ciudadanos, vanguardistas, Nicholas Tomaiuolo (2009) distingue medios que admiten contenidos producidos por usuarios, como CNN (I report), BBC Have your say, CBS Eye mobile, Msnbc Citizen 


\begin{tabular}{|l|c|c|c|c|r|r|}
\hline \multirow{2}{*}{ Herramientas } & \multicolumn{2}{c|}{ Periódicos españoles (\%) } & \multicolumn{2}{c|}{ Periódicos norteamericanos (\%) } \\
\cline { 2 - 7 } & $\mathbf{2 0 0 6}$ & $\mathbf{2 0 0 8}$ & Dif. & $\mathbf{2 0 0 6}$ & $\mathbf{2 0 0 8}$ & Dif. \\
\hline RSS & 23,5 & 66,7 & 43,2 & 76 & 100 & 24 \\
\hline Más popular & 22,4 & 55,6 & 33,2 & 33 & 76 & 43 \\
\hline Vídeo & 23,5 & 58,9 & 35,4 & 61 & 100 & 39 \\
\hline Podcasts & 3,5 & 3,3 & $-0,2$ & 31 & 40 & 9 \\
\hline Blog de reporteros & 31,8 & 58,9 & 27,1 & 80 & 94 & 14 \\
\hline Comentarios en blogs & 29,4 & 58,9 & 29,5 & 67 & 92 & 25 \\
\hline Comentarios en noticias & 10,6 & 50,0 & 39,4 & 19 & 75 & 56 \\
\hline Marcadores sociales & 2,4 & 54,4 & 52,0 & 7 & 92 & 85 \\
\hline
\end{tabular}

Fuente: García-De-Torres et al. (2008)

Tabla 1. Evolución de las herramientas 2.0 en los diarios españoles y norteamericanos (para la muestra de los españoles se tomaron 85 diarios en 2006 y 90 en 2008, y para los norteamericanos 100 diarios del Bivings Group Study, tanto en 2006 como en 2008)

journalist, The New York times (The local), Fox news (U report), Newsvine y Yahoo you witness news; en segundo lugar, sitios de ámbito local que admiten contribuciones supervisadas en la mayoría de los casos por periodistas profesionales, como Chi-Town daily news, Citizen journalism (Washington times), ITowns (Hartford courant), MinnPost, New West; por último, sitios tradicionales que admiten interacción marginal como Times people (The New York times).

Las razones son diversas (Vujnovic et al., 2010). Un estudio sobre la actitud de los editores hacia el CGU en 29 publicaciones de Texas (Lewis et al., 2010) pone de manifiesto que si bien aceptan mayoritariamente el CGU, sólo la mitad de ellos lo hace porque promueven un mayor compromiso cívico o participación y constituye un suplemento; pesa el componente práctico. Los que objetan, lo hacen con referencia a que es una profesión que no puede desempeñar cualquier persona, al desconocimiento de la ética y de las rutinas profesionales, al trabajo que supone la comprobación, al desconocimiento de las consecuencias legales por parte de los usuarios y al peso de la opinión frente a los hechos.

En los medios, las opciones de participación son mayores en torno a los contenidos elaborados por los profesionales de cada medio (Domingo et al., 2008; García-De-Torres et al., 2009; Lacy et al., 2010). Franck Rebillard y Annelise Touboul (2010) sostienen que la verificación empírica de los cambios en la participación -siendo que ésta se mantiene en niveles bajos- no apoya

\begin{tabular}{|l|c|c|}
\hline $\begin{array}{c}\text { Tipo de información que } \\
\text { puede subirse }\end{array}$ & $\begin{array}{c}\text { Diarios } \\
\mathbf{n = 6 3}\end{array}$ & $\begin{array}{c}\text { Sitios de } \\
\text { periodismo } \\
\text { ciudadano } \\
\text { (n=53) }\end{array}$ \\
\hline sobre actividades comunitarias & 67 & 70 \\
\hline noticias o reportajes & 44 & 62 \\
\hline cartas al editor & 64 & 40 \\
\hline audio & 3 & 28 \\
\hline fotografías & 49 & 45 \\
\hline vídeo & 24 & 34 \\
\hline Fuente: Elaborado a partir de Lacy et al., 2010
\end{tabular}

Tabla 2. Opciones de publicación en diarios y medios ciudadanos la tesis de la revolución digital. Además, diversos estudios han puesto de manifiesto la incapacidad de los usuarios de contribuir al debate público (Díaz-Noci, 2010; Acosta, 2008). Rost, Pugni-Reta y Apesteguía (2008) han estudiado las causas de la abstención de los usuarios, aportando una perspectiva imprescindible para evaluar adecuadamente la participación de la gente en los medios, que apuntan a desconfianza hacia el medio y los periodistas, desinterés personal por el debate, dificultad tecnológica y falta de interés del propio medio. En el nivel macro-social amplio, el impacto puede ser mayor. Algunos blogs superan a grandes medios (Calmon-Alves, 2008) y el ecosistema informativo -no la agenda- ha experimentado una transformación profunda (Kovach y Rosenstiel, 1999).

\section{"La investigación sobre contenidos generados por el usuario se inició en los años noventa"}

\section{Investigación sobre contenidos generados por el usuario}

La investigación sobre CGU se inicia en los años noventa y se centra principalmente en el desarrollo de opciones de participación en los medios, por lo que tiene larga tradición en un campo de investigación joven.

El impulso inicial fue notable (Neuberger, Tonnemacher, Biebl y Duck, 1998; Light y Rogers, 1999; Massey y Levy, 1999; Shultz, 1999; Kenney, Gorelik y Mwangi, 2000; Paulussen, 2002). No olvidemos que apenas unos años antes Merril Morris y Christine Ogan (1996) exponen que, al conceptualizar internet como medio de comunicación de masas, resulta claro que ni "masivo" ni "medio" pueden ser definidos con claridad para todas las situaciones y que el estudio de internet como medio de comunicación de masas implica la reconceptualización de la audiencia, la credibili- 
dad y el intercambio de productores y receptores.

Las primeros estudios tenían limitaciones. Las herramientas interactivas, sostiene Tanjev Shultz (1999), son una condición necesaria para el inicio de un discurso interactivo, pero la mera presencia o disponibilidad dice poco sobre cómo las utilizan las audiencias y los periodistas. Kenney, Gorelik y Mwangi (2002) señalan dos líneas "evidentes" de investigación para el futuro: entrevistar a ejecutivos de periódicos online (para saber la razón por la cual las herramientas de interactividad no son usadas de forma más extensa) y explorar la experiencia de los usuarios.

En la medida en que el contenido generado por el usuario se amplía y diversifica, se hace patente que el marco de análisis de un canal híbrido (Reardon y Pillis, 1996; Secko, 2009) requiere la integración de teorías y modelos de flujos alocutorios, conversacionales, de registro y de consulta, así como de los campos de la producción y la recepción, dos áreas de la investigación en comunicación de masas que convergen en el CGU.

La observación y el cuestionario, más habituales al principio en estudios de carácter cuantitativo, han dado paso a metodologías cualitativas y enfoques triangulares, con incremento de la entrevista en profundidad y el estudio etnográfico (Boczkowski, 2005; Robinson, 2010).

Asimismo, abordajes generalistas han dado paso a análisis de aspectos concretos relacionados con el contenido generado por el usuario, entre los que destacan la calidad del CGU y las prácticas de crowdsourcing, el impacto en las rutinas de los medios o el control y el reconocimiento de las contribuciones de los usuarios, especialmente en los Estados Unidos.

En América Latina, Rost ha contribuido a desarrollar el concepto de interactividad y ha seguido la evolución de la participación en los medios argentinos -Rost y Pugni-Reta (2005), Rost (2006, 2007, 2008 y 2010) y Rost, Pugni-Reta y Apesteguía (2008), así como García (2007) e Igarza (2009 y 2009a)-. Podemos mencionar asimismo, los estudios sobre interactividad en los medios mexicanos de Caballero (1998, 2000), Navarro (2003, 2004, 2009), López-Aguirre (2009) y Lerma-Noriega (2009). En Perú -donde es pionera Rosa Zeta-De-Pozo (2002) - encontrarmos referencias a la participación en los trabajos de Lyudmyla get reports: RSS | KML | JSON | CSV (table)

\section{Oil Spill Crisis Map}

(2)

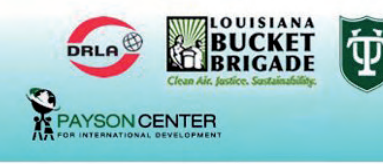

SUEMIT AN INGIDENT GETALERTS CONTACTUS RESOURCES ABOUTUS

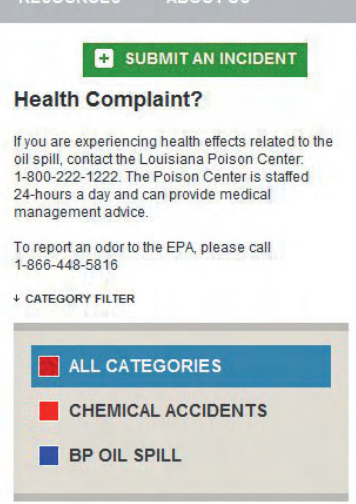

(2)

+ OTHER LAYERS

FOSSIL FUEL AND POWER PLANTS

HAZARDOUS WASTE

CHEMICAL PLANTS

REFINERIES

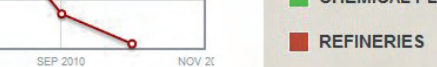

(1)

Figura 3. Ciudadanos periodistas organizados por la Lousiana Bucket Brigade ayudaron a apa sobre el vertido de petróleo en el Golfo de México http://oilspill.labucketbrigade.org/

Yezers'ka (2008, 2008a). También en un contexto más amplio, aborda la interactividad en los medios venezolanos Cely Rojano (2006). En Colombia, y de manera monográfica, han publicado análisis de opciones o rasgos de la participación de los usuarios Llano (2005), Alonso-Sánchez (2008) y Zúñiga y Duque (2009).

Cuatro trabajos tienen en cuenta un espectro más amplio de países. Guillermo Franco y Julio-César Guzmán $(2004,2007)$ han examinado las herramientas de interactividad en un amplio grupo de diarios de referencia de América Latina y dos estudios comparativos han abordado los escenarios de España, México, Perú y Venezuela (García-De-Torres y Rodríguez-Martínez, 2007) y Argentina, Colombia, México, Perú, Estados Unidos, España, Portugal y Venezuela (GarcíaDe-Torres et al., 2009).

\section{"Crece el interés por la oportunidad del CGU en los modelos de negocio y, en lo legal, por los derechos de autor"}

Neil Thurman es pionero y ha impulsado la investigación en este ámbito en Europa tanto en cuanto 


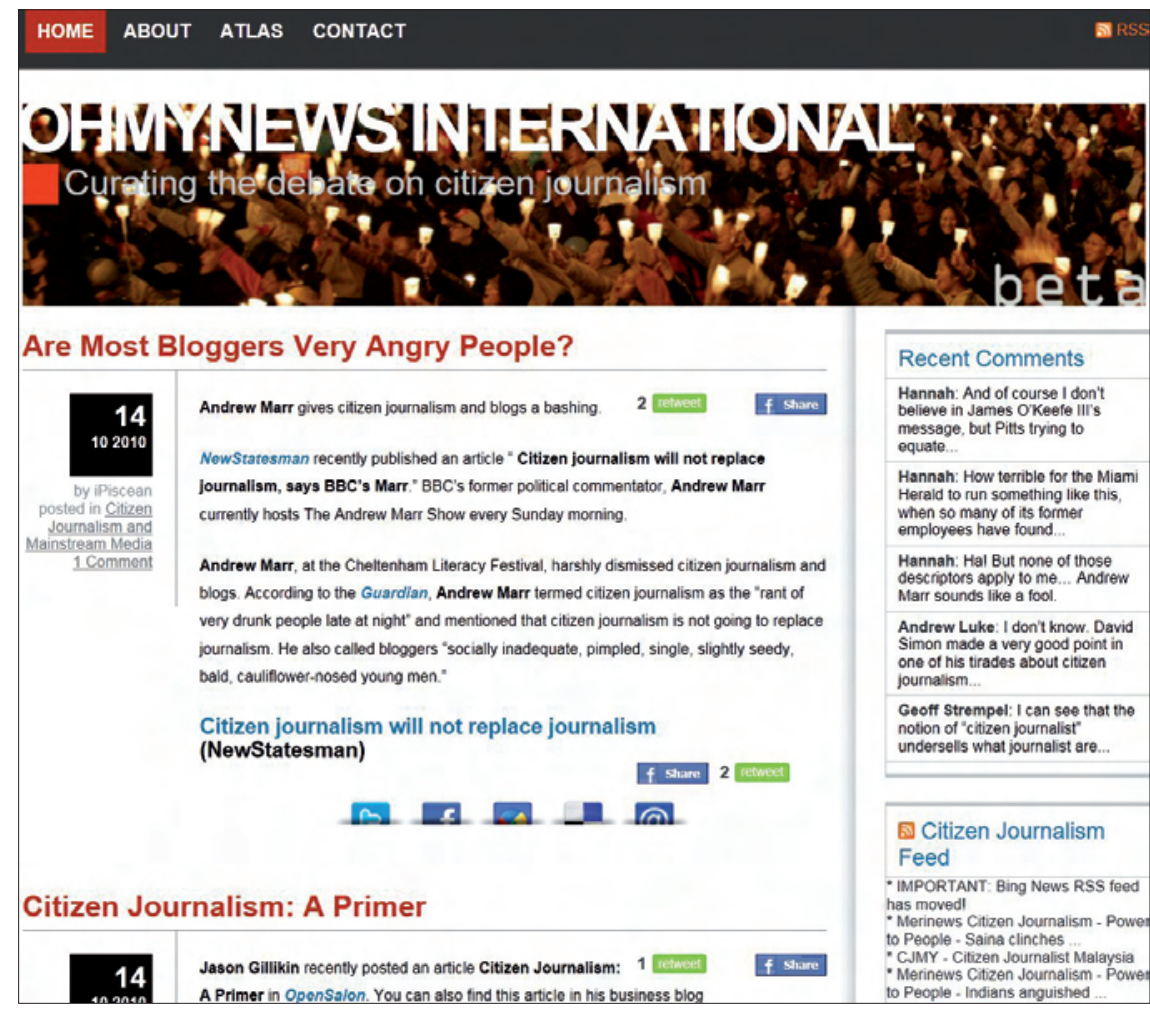

Figura 4. OhMyNews International se reconvierte en blog, ante la dificultad de gestionar con suficiente calidad la información ciudadana http://international.ohmynews.com/

a renovación metodológica como a la orientación de los estudios. Podemos mencionar contribuciones en los escenarios portugués (Zamith, 2008 y 2008a), francés (Jouet, 2009; Rebillard y Touboul, 2010), británico (Thurman, 2006; Thurman y Herbert, 2007; Thurman y Hermida, 2008, Singer y Ashman, 2009; Thurman y Hermida, 2010; Singer, 2010; Harrison, 2010), griego (Spyridou, 2008), alemán (Gerpott y Wanke, 2004), español (Domingo, 2008; García-DeTorres, 2006; García-De-Torres et al., 2008a; GarcíaDe-Torres et al., 2009a; Meso-Ayerdi y Palomo, 2009; Martínez-Martínez, 2009; Noguera, 2010, Díaz-Noci et al., 2010), de los Países Bajos (Paulussen y Ugille, 2008; De-Kayser y Raemecker, 2008; Bäkker y Mervi, 2009) y Eslovenia (Oblak, 2005; Kovacic y Erjavec, 2008). Existe una notable tradición de estudios comparativos, con el antecedente de Fortunati et al. (2005): Deuze, Bruns y Neuberger (2007), Paulussen, Heinonen, Domingo y Quandt (2007), Domingo et al. (2008), Örnebring (2008) y Vujnovic et al. (2010).

En la investigación sobre el CGU sobre los medios de Estados Unidos en los últimos años se aborda de manera pionera la calidad y la credibilidad del CGU, así como su integración en las prácticas periodísticas. Destacan las aportaciones de Lawrey y Anderson (2005), Zeng y Li (2006), MacGregor (2007), Bradshaw (2007), Johnson (2007), Reich (2008), Abdul-Mageed (2008), Gunter et al. (2009), Chung (2009), Johnson y Wiedenbeck (2009), Muthukumaraswamy (2010), Lacy et al. (2010), Lewis et al.
(2010), Robinson (2010) y Berger y Milkman (2010).

En otros continentes la producción es menor y es más difícil, por tanto, hacer una aproximación regional al CGU (Folayan, 2004; Xiao y Polumbaum, 2006; Hashim, Hasan y Sinnapan, 2007; Rahman, 2008; Foster, 2008; Allan y Thorsen, 2009; Xu, 2009; Haneefa y Nellikka, 2010; Stassen, 2010). Recientemente, Bergland et al. (2010) han analizado la interactividad en los diarios de Estados Unidos, Canadá, Gran Bretaña, Nueva Zelanda y Australia.

Los enfoques multidisciplinares son cada vez más necesarios, tanto por la repercusión del CGU en los modelos de negocio (Herrero-Subías, 2008; Rojo-Villada，2008; Nieto, 2008; Picard, 2009; Cea, 2009; Larrañaga, 2009; Graham et al., 2010) como por las derivaciones legales. Aunque la investigación se centra preferentemente en la protección de los derechos de autor, en los últimos años, se tiende a una revisión crítica de las cuestiones que atañen al derecho en el contexto de las tecnologías 2.0: Milstein (2007), Latham, Butzer y Brown (2008), Boortz (2008), Montagnani (2009), Corredoira (2009), Meyer (2009) y Lee (2009), entre otros.

\section{"En la investigación reciente destacan los estudios sobre la calidad del CGU y las prácticas de crowdsourcing"}

\section{Conclusiones}

Más de diez años después de la inauguración de los estudios sobre interactividad en los medios informativos digitales se aprecia un recorrido desigual por regiones, y planteamientos avanzados metodológicamente que conviven con aproximaciones que reproducen los esquemas de análisis de los primeros trabajos, en un campo en el que destacan los estudios comparativos.

Los investigadores han asumido el reto de dar cohesión a las diferentes áreas de estudio de la comunicación, que convergen en el estudio del contenido generado por el usuario. Los hallazgos en producción y 
recepción en el ámbito de la comunicación de masas convergen en la formulación tanto del marco teórico como de la metodología en las investigaciones más recientes.

Menos se ha avanzado, en cambio, en la definición del canal híbrido que es internet y las consecuencias de la integración de modos de comunicación mediada y de masas, algo fundamental cuando se aborda la comunicación a través de internet en combinación con dispositivos móviles a través de los cuales se dinamiza la participación ciudadana.

Más allá de constatar la presencia de contenidos generados por los usuarios, los investigadores se adentran ahora en un camino difícil pero necesario para comprender cómo será el periodismo del futuro: evaluar la calidad de los contenidos que aportan los usuarios. La sistematización de los hallazgos con una perspectiva global, la multidisciplinariedad y el trabajo de clarificación conceptual son algunos retos para el futuro de la investigación en este campo.

\section{Bibliografía citada}

Abdul-Mageed, Muhammad M. "Online news sites and journalism 2.0. Reader comments on Al Jazeera Arabic". 2008 triple C 6, n. 2, pp. 59-76.

Acosta-Valencia, Gladys L. "Relación dialógica o comunicación paranoica. Un análisis de lo que acontece en los foros del lector que promueve la prensa digital en Colombia". $10^{\circ}$ Congreso Redcom. Conectados, hipersegmentados y desinformados en la era de la globalización, 2008, Salta. http://www.ucasal.net/unid-academicas/artes-y-ciencias/congresos/ redcom10/archivos/redcom-ponencia/Eje5/Mesa5-1/Acosta-Valencia.pdf

Allan, Stuart; Thorsen, Einar. Citizen journalism. Global perspectives. New York: Peter Lang Publishing, 2009. ISBN 1947-2587.

Bäkker, Piet; Pantti, Mervi. "Beyond news. User-generated content on Dutch media websites". Future of journalism conf, 2009. Cardiff.

Berger, Jonah A.; Milkman, Katherine L. "Social transmission, emotion, and the virality of online content", 2009.

http://ssrn.com/abstract $=1528077$

Bergland, Robert; Crawford, Lisa; Noe, Sarah; Hon, David. "Multimedia and interactivity on newspaper websites. A multi-study analysis of seven English-speaking countries". 10 Intl online journalism symp, 2010, Univ de Texas, Austin.

http://online.journalism.utexas.edu/2010/papers/Berglandetal10.pdf

Blood, Rebecca. We've got blog. Cambridge: Perseus Publishing, 2002.

Boczkowski, Pablo J. Digitizing the news. Innovation in online newspapers. Boston, MA: The MIT Press, 2005.

Bowman, Shayne; Willis, Chris. We media. How audiences are shaping the future of news and information. Reston: The Media Center at the American Press Institute, 2002.

http://www.hypergene.net/wemedia/download/we_media.pdf

Bradshaw, Paul. "Wiki journalism: are wikis the new blogs?". Future of newspaper conf, 2007, Cardiff.

http://onlinejournalismblog.files.wordpress.com/2007/09/wiki_journalism. $p d f$

Caballero, Uriel. "El periodismo impreso mexicano en busca del ciberespacio". Revista mexicana de comunicación, 1998, n. 55.

http://www.cem.itesm.mx/dacs/buendia/rmc/rmc55/uriell.html

Caballero, Uriel. "Periódicos mexicanos en internet". Revista Univ de Guadalajara, 2000, n. 22.

http://www.cge.udg.mx/revistaudg/rug22/rug22dossier3.html

Calmon-Alves, Rosental. "Tendências do jornalismo para a emergente sociedade em rede". I Congresso intl de ciberjornalismo, 2008. Oporto.
Cea, María-Nereida. "Modelo de negocio de la empresa periodística en internet. El caso de Prisa". Revista latina de comunicacion social, 2009, n. 64 .

http://www.revistalatinacs.org/09/art/872_UMA/73_99_Nereida_Cea_ Esteruelas.html

Chung, Deborah S. "How readers perceive journalists' functions at online community newspapers". Newspaper research journal, 2009, v. 30, n. 1, pp. 72-80.

Corredoira, Loreto. "Diarios on line. Consecuencias legales del periodismo en la web 2.0". Informe AEDE sobre la prensa diaria, 2008.

Deuze, Mark. "Participation, remediation, bricolage: considering principal components of a digital culture". The information society 2006, v. 2, n. 22, pp. 63-75.

Deuze, Mark; Bruns, Axel; Neuberger, Christoph. "Preparing for an age of participatory news". Journalism practice, 2007, v. 1, n. 3, pp. 322-338.

Díaz-Noci, Javier; Domingo, David; Masip, Pere; Micó-Sanz, JosepLluís; Ruiz, Carles. "Comments in news, democracy booster or journalistic nightmare. Assessing the quality and dynamics of citizen debates in Catalan online newspapers". 10 $0^{\text {th }}$ Intl online journalism Symp, 2010, Univ de Texas, Austin.

Domingo, David. "Interactivity in the daily routines of online newsrooms. Dealing with an uncomfortable myth". Journal of computer-mediated communication, 2008, v. 13, n. 3, pp. 680-704.

Domingo, David; Quandt, Thorsen; Heinonen, Ari; Paulussen, Steve; Singer, Jane B.; Vujnovic, Marina. "Participatory journalism practices in the media and beyond. An international comparative study of initiatives in online newspapers". Journalism practice, 2008, v. 2, n. 3, pp. 326-342.

Folayan, Oluseyi-Olukemi. Interactivity in online journalism. A case study of the interactive nature of Nigeria's online Guardian. Masters thesis, Rhodes Univ, 2004.

Font, José-Luis. "La prensa latinoamericana en internet". Sala de prensa, 1999, año II, v. 2.

http://www.saladeprensa.org

Fortunati, Leopoldina; Raycheva, Lilia; Harro-Loit, Halliki; O’Sullivan, John. "Online news interactivity in four European countries. A pre-political dimension”. Informació on line, 2005, v. 1, n. 5, pp. 417-429.

Foster, Sophie. "Journalism in the new age of participation. Meeting the challenge in Fiji newsrooms". Fijian studies. A journal of contemporary Fiji, 2008, v. 6, n. 1-2, pp. 112-129.

Franco, Guillermo; Guzmán, Julio-César. "Situación del periodismo digital y de los sitios web".

https.//www.poynter.org/content/content_view.asp?id=124673

Franco, Guillermo; Guzmán, Julio-César. "Encuesta sobre periodismo de internet en Latinoamérica". Sala de prensa, 2004, v. VI, n. 3.

http://www.saladeprensa.org/art551.htm

García-De-Torres, Elvira; Rodríguez-Martínez, Janet. "Herramientas web 2.0 en los diarios digitales de España, Venezuela, Perú y México". En: Sabés, F. (coord.): Presente y futuro de la comunicación digital. Huesca: Asociación de la Prensa de Aragón, 2007.

García-De-Torres, Elvira; Rodríguez-Martínez, Janet; Martínez-Martínez, Silvia; Ruiz-Grau, Silvana; Albacar, Helena. "Tejiendo la red informativa". En: IX Congreso latinoamericano de investigación en la comunicación (Alaic), 2008, Instituto Tecnológico de Monterrey, México.

García-De-Torres, Elvira; Saiz-Olmo, Jesús; Rodríguez-Martínez, Janet; Martínez-Martínez, Silvia; Ruiz-Grau, Silvana; Albacar, Helena. "Las herramientas 2.0 en los diarios españoles 2006-2008". Prisma.com, 2008a, n. 6.

http://prisma.cetac.up.pt/edicao_n7_dezembro_de_2008/las_herramien tas_20_en_los_dia.html

García-De-Torres, Elvira; Martínez-Martínez, Silvia; Cebrián, Bernardino; Rodríguez-Martínez, Janet; Albacar, Helena. "La agenda de los usuarios. Un análisis de la participación en la página principal y la sección local de Elpais.com y 20minutos.es". En: Herrero, P.; Rivas, P.; Gelado, R.: Estudios de periodística XIV, Periodismo ciudadano, posibilidades y riesgos para el discurso informativo, 2009, pp. 287-300.

García-De-Torres, Elvira; Yerzers'ka, Lyudmyla; Rojano, Miladys Igarza, Roberto; Azevedo, José; Zamith, Fernando; Paul, Nora; Calderín, Mabel; Sánchez-Badillo, Jorge; Martínez-Martínez, Silvia; Rodríguez-Martínez, Janet. "UGC status and levels of control in Argentinean, 
Colombian, Mexican, Peruvian, Portuguese, Spanish, US and Venezuelan online newspapers". 10 $0^{\text {th }}$ Intl symp on online journalism, 2009a, Univ de Texas, Austin

http://online.journalism.utexas.edu/2009/papers/Garciaetal09.pdf

García-Orosa, Berta; Capón-García, José-Luis. "Las bitácoras o weblogs y la lógica del campo informativo. Un análisis comparativo con la agenda mediática tradicional". Estudios sobre el mensaje periodístico, 2004, n. 10, pp. 113-128.

García, Edgardo-Pablo. "Interactivity in Argentinean online newsrooms". Zer English edition, 2007, v. 1, pp. 7-25.

http://www.ehu.es/zer/zere1/ZERE_epgarcia.pdf

Gerpott, Thorsten J.; Wanke, Hendrik. "Interactivity potentials and usage of German press-title web sites. An empirical investigation". Journal of media economics, 2004, v. 17, n. 4, pp. 241-260.

Gillmor, Dan. "We the media. Grassroots journalism by the people, for the people”. Sebastopol: Ed. O'Reilly, 2004.

Graham, Gary; Kerrigan, Finola; Mehmood, Rashid; Rahman, Mustafizur. "The interaction of production and consumption in the news media". Lecture notes of the Institute for Computer Sciences, Social Informatics and Telecommunications Engineering, 2009, v. 16, n. 4, pp. 229-239.

Gunter, Barrie; Campbell, Vincent; Touri, Maria; Gibson, Rachel. "Blogs, news and credibility". Aslib proc, 2009, v. 61, n. 2, pp. 185-204.

Haneefa, Mohamed; Nellikka, Shyma. "Content analysis of online English newspapers in India". Journal of library \& information technology, 2010, v. 30, n. 4, pp. 17-24.

Harrison, Jackie. "User-generated content and gatekeeping at the BBC hub". Journalism studies, 2010, v. 11, n. 2, pp. 243-256.

Hashim, Lina; Hasan, Helen; Sinnapan, Suku. "Australian online newspapers. A website content analysis approach to measuring interactivity". $18^{\text {th }}$ Australasian conf on information systems (ACIS), 2007, Toowoomba. http://ro.uow.edu.au/cgi/viewcontent.cgi? article $=1435 \&$ context $=$ commpapers

Hermida, Alfred; Thurman, Neil. "A clash of cultures. The integration of user-generated content within professional journalistic frameworks at British newspaper websites". Journalism practice, 2008 , v. 2, n. 3, pp. 343356.

Hermida, Alfred. "The blogging BBC. Journalism blogs at 'the world's most trusted news organisation". Journalism practice, 2009, v. 3, n. 3, pp. $1-17$

Igarza, Roberto. "La fruición participativa". En: Burbujas de ocio. Nuevas formas de consumo cultural, pp. 177-181. Buenos Aires: La Crujía, 2009.

Igarza, Roberto. "Nuevos medios, nuevos modos, nuevos lenguajes". En: Irigaray, Fernando; Ceballos, Dardo; Manna, Matías: Periodismo participado en el paradigma de la transición, 2009a, pp. 165-182. Univ Nacional de Rosario.

http://www.scribd.com/doc/19540523/Nuevos-medios-nuevos-modosnuevos-lenguajes-1er-Foro-de-Periodismo-Digital-de-Rosario-2008

Islas, Octavio; Gutiérrez, Fernando. "Teorías e investigación de internet en México". En: Lozano Rendón, J. C.: La comunicación en México Diagnóstico, balances y retos. Monterrey: Coneicc-Itesm, 2005.

Johnson, Kirsten A. "The impacts of hyperlinks and writer information on the credibility of stories on a participatory journalism web site". En: Baranauskas, C.; Palanque, P.; Abascal, J.; Barbosa, S.D.J.: Human computer interaction 2007. Lectures notes in computer Science, 2010, v. 4663, pp. 580-585.

Johnson, Kirsten A.; Wiedenbeck, Susan. "Enhancing perceived credibility of citizen journalism web sites». J\&MC quarterly, 2009, v. 86, n. 2, pp. 332-348.

Kenney, Keith; Gorelik, Alexander; Mwangi, Sam. "Interactive features of online newspapers". First Monday, 2000, v. 5, n. 1. http://www.firstmonday.dk/issues/issue5_1/kenney/

Keshvani, Nisar. "Trends in the online newsroom. A study of the Straits times interactive", 2000

http://www.keshvani.com/academia/apmeonlinenews_092000.pdf

Keyser, Jeroen; Raeymaeckers, Karin. "Forcing the gates of the fortress The growing impact of public participation in Flemish newsrooms". Tech nology, education and ethics conf, 2008.

http://theendofjournalism.wdfiles.com/local--files/dekeyseretal/De\%20Keys er\%20\&\%20Raeymaeckers.pdf
Kovach, Bill; Rosenstiel, Tom. Warp speed: America in the age of mixed media. Nueva York: Century Foundation Book, 1999.

Kovacic, Melita-Poler; Erjavec, Karmen. "Mobi journalism in Slovenia. Is this really citizen journalism?". Journalism studies, 2008, v. 9, n. 6, pp. $874-890$

Lacy, Stephen; Duffy, Margaret; Riffe, Daniel; Thorson, Esther; Fleming, Ken. "Citizen journalism web sites complement newspapers". Newspaper research journal, 2010, v. 31, n. 2, pp. 34-46.

Larrañaga, Julio. "La crisis del modelo económico de la industria de los periódicos". Estudios sobre el mensaje periodístico, 2009, n. 15, pp. 61-80.

Latham, Robert P.; Butzer, Carl C.; Brown, Jeremy T. "Legal implications of user-generated content: YouTube, MySpace, Facebook". Intellectual property \& technology law journal, 2008, v. 20, n. 5, pp. 1-11.

Lee, Edward. "Developing copyright practices for user-generated content". Journal of internet Law, 2009, v. 13, n. 1, pp. 1-23.

Lerma-Noriega, Claudia-Alicia. La usabilidad de la prensa digital de habla hispana. Análisis de los sitios web de ABC, El Mundo, El Universal y La Jornada. Tesis doctoral dirigida por Roberto De-Miguel-Pascual. Fac. de Ciencias de la Información, Univ Complutense de Madrid, 2004.

Lerma-Noriega, Claudia-Alicia. "Implementación del periodismo interactivo en los portales de la prensa mexicana”. Revista latina de comunicación social, 2009, n. 64, pp. 787-801.

http://www.revistalatinacs.org/09/art/861_ITESM/62_68_CA_Lerma.html

Lewis, Seth; Kawfhold, Kelly; Lasorsa, Dominic L. "Thinking about citizen journalism. The philosophical and practical challenges of user-generated content for community newspapers". Journalism practice, 2010, v. 4, n. 2, pp. 163-179.

López-Aguirre, José-Luis. Estudio de servicios de e-comunicación y productos ciberperiodísticos en la prensa digital mexicana 2008, 2009. http://www.ucm.es/BUCM/revistas/byd/11321873/articulos/RGID 0909110029A.PDF

Light, Ann; Rogers, Yvonne. "Conversation as publishing. The role of news forums on the Web". Journal of computer mediated communication, 1999, v. 4, n. 4

http://www.ascusc.org/jcmc/vol4/issue4/light.html

Llano, Sergio. "Hipermedia e interactividad en el periodismo digital colombiano". Palabra clave, 2005, n. 12.

http://redalyc.uaemex.mx/redalyc/pdf/649/64901205.pdf

Mac Síthigh, Daithí. "The mass age of internet law". Information \& communications technology law, 2008, v. 17, n. 2, pp. 79-84.

MacGregor, Phil. "Tracking the online audience". Journalism studies, 2007 , v. 8 , n. 2 , pp. $280-298$

Maletzke, Gerhard. Sicología de la comunicación. Quito: Ciespal, 1963.

Martínez-Martínez, Silvia. "Nota de investigación: la interactividad en los diarios digitales españoles (2006-2008)". Doxa comunicación, 2008, n. 7, pp. 157-164.

http://www.humanidades.uspceu.es/pdf/Notainvest1Lainteractividad.pdf

Massey, Brian L.; Levy, Mark R. "Interactive online journalism at Englishlanguage web newspapers in Asia”. Gazette, 1999, v. 61, n. 6, pp. 523-538.

Meso-Ayerdi, Koldobika; Palomo-Torres, María-Bella. "Periodismo ciudadano, posibilidades y riesgos para el discurso informativo. En: PérezHerrero, Pilar, Rivas-Nieto, Pedro; Gelado-Marcos, Roberto: Estudios de periodística XIV, 2009, pp. 253-262. ISBN 978-84-7299-842-1.

Milstein, Lee C. "Avoiding legal pitfalls on user-generated content sites". Journal of internet law, 2007, v. 11, n. 3, pp. 3-10.

Montagnani, Maria-Lilla. "A new interface between copyright law and technology. How user-generated content will shape the future of online distribution". Cardozo arts \& entertainment law journal, 2009, v. 26, n. 3, pp. 719-773.

Markovits, Stefanie. 'Rushing into print: 'participatory journalism' during the Crimean War”. Victorian studies, 2008, v. 50, n. 4, pp. 559-586.

Morris, Merrill; Ogan, Christine. "The internet as mass medium". Journal of communication, 1996, v. 46, n. 1, pp. 39-50.

Muthukumaraswamy, Karthika. "When the media meet crowds of wisdom. How journalists are tapping into audience expertise and manpower for the processes of newsgathering". Journalism practice, 2010, v. 4, n. 1, pp. 48-65. 
Navarro-Zamora, Lizy. "1994-2004. Diez años del periodismo on line". Estudios sobre el mensaje periodístico, 2004, n. 10, pp. 159-174.

Navarro-Zamora, Lizy. "El periodismo on-line en México". Razón y palabra, 2003, n. 30.

http://www.cem.itesm.mx/dacs/publicaciones/logos/anteriores/n30/ lnavarro.html

Navarro-Zamora, Lizy. "Tres lustros del periodismo digital. interactividad e hipertextualidad". Comunicar, 2009, v. XVII, n. 33, pp. 35-43.

Neuberger, Christoph; Tonnemacher, Jan; Biebl, Matthias; Duck, André. "Online - the future of newspapers? Dailies on the world wide web". Journal of computer mediated communication, 1998, v. 4, n. 1.

http://www.ascusc.org/jcmc/vol4/issue1/neuberger.html

Newhagen, John E.; Levy, Mark R. "Distributed communication architectures and news". En: The future of jornalism in a distributed communication architecture, 1996.

http://jnews.umd.edu/johnen/research/grape.html

Newhagen, John E.; Rafaeli, Sheizaf. "Why communication researchers should study the internet. A dialogue". Journal of communication, 1996, v. 46, n. 1, pp. 4-13.

Nieto, Alfonso. "Ciudadano y mercado de la comunicación". Comunicacion y sociedad, 2008, v. 21, n. 2, pp. 7-33.

Noguera, José-Manuel. "Redes sociales como paradigma periodístico. Medios españoles en Facebook". Revista latina de comunicación social, 2010, n. 65, pp. 176-186.

http://www.revistalatinacs.org/10/art/891_UCAM/13_JM_Noguera.html

Oblak, Tanja. "The lack of interactivity and hypertextuality in online media". Gazette, 2005, v. 67, n. 1, pp. 87-106.

Örnebring, Henrik. "The consumer as producer - of what? User-generated tabloid content in The Sun (UK) and Aftonbladet (Sweden)». Journalism studies, 2008, v. 9, n. 5, pp. 771-785.

Outing, Steve. "The 11 Layers of citizen journalism. A resource guide to help you figure out how to put this industry trend to work for you and your newsroom", 2005.

http://www.poynter.org/content/content_view.asp? $i d=83126$

Paterson, Chris; Domingo, David. Making online news. The ethnography of new media production. New York: Peter Lang, 2008.

Paulussen, Steve; Heinonen, Ari; Domingo, David; Quandt, Thorsten. "Doing it together. Citizen participation in the professional news making process". $O B S, 2007$, v. 1, n. 3, pp. 131-154.

Paulussen, Steve. "Online journalism and new media in Flanders". New media and old media COST Conference, 2002. Troms $\emptyset$ (Noruega). http://cost-a20.iscte.pt/tromso/Online_journalism_Flanders.txt

Paulussen, Steve; Ugille, Pieter. "User generated content in the newsroom. Professional and organisational constraints in participatory journalism". Westminster papers in communication and culture, 2008, v. 5, n. 2, pp. 24-41.

Pew Research Institute. Understanding the Participatory News Consumer How internet and cell phone users have turned news into a social experience. Pew Research Center's Project for Excellence in Journalism, 2010. http://www.journalism.org/analysis_report/understanding_participatory_ news_consumer

PEJ Report 2009. "On online community journalism sites- Phase II”. http://www.stateofthenewsmedia.org/2009/chapter\%20pdfs/PewKnightrepo $r t \% 2008 \% 20 F I N A L . p d f$

Picard, Robert G. "Blogs, tweets, social media, and the news business". Nieman reports, 2009.

http://www.nieman.harvard.edu/reportsitem.aspx?id=101884

Pinto, Timothy; Shan, Niri; Freytag, Stefan; Braunschweig, Elisabeth Aumage, Valerie. "Liability of online publishers for user generated content. A European perspective". Communications lawyer, 2010, v. 27, n. 1, pp. 5-13.

Quandt, Thorsten. "(No) news on the world wide web?". Journalism studies, 2008, v. 9, n. 5, pp. 717-738.

Quiroga, Evangelina. "La participación de los públicos en los medios. De las cartas de los lectores a los contenidos informativos generados por los usuarios". En: $10^{\circ}$ Congreso Redcom, 2008. Salta.

Rahman, Mashihur. "Interactive options in online newspapers of Bangladesh". Pakistan journal of social sciences, 2008, v. 5, n. 6, pp. 620-624.
Reardon, Kathleen; De-Pillis, Emmeline G. "Multichannel leadership. Revisiting the false dichotomy". En: An integrated approach to communication theory and research, pp. 399-408. New Jersey: Laurence Earlboum, 1996.

Rebillard, Franck; Touboul, Annelise. "Promises unfulfilled "Journalism 2.0" user participation and editorial policy on newspaper websites". Media, culture \& society, 2010, v. 32, n. 2, pp. 323-334.

Restrepo, María-Teresa; Herran, Javier-Darío. Ética para periodistas. Bogotá: Tercer Mundo Editores, 1991.

Robinson, Sue. "Traditionalists vs. convergers". Convergence: The international journal of research into new media technologies, 2010, v. 16, n 1, pp. 125-143.

Rojano, Miladys (coord.). Diez años de periodismo digital en Venezuela 1996-2006. Caracas: Univ Católica Andrés Bello, 2006.

Rojas-Orduña, Octavio; Alonso, Julio; Orihuela, José-Luis; Antúnez, José-Luis; Varela, Juan. Blogs. La conversación en internet que está revolucionando medios, empresas y ciudadanos. Madrid: Esic, 2005.

Rojo-Villada, Pedro-Antonio. "El acceso a la información en la era digital. Situación actual y perspectivas desde las empresas periodísticas multimedia”. Comunicación, 2008, v. 1, n. 6, pp. 159-172.

Rost, Alejandro. "La participación en el periodismo digital. Muchas preguntas y algunas posibles respuestas". En: Irigaray, Fernando; Ceballos, Dardo; Manna, Matías: Periodismo digital en un paradigma en transición. Univ Nacional de Rosario, 2010, pp. 96-109.

Rost, Alejandro. "Hipervínculos y opciones de participación en Clarín. com". En: Periodismo digital en Argentina. Diseño, interactividad, hipertexto y multimedialidad en sitios de noticias, 2008, pp. 97-125.

Rost, Alejandro; Pugni-Reta, María-Emilia; Apesteguía, Ezequiel "Cómo navegan los usuarios de sitios de noticias". En: Bergonzi et al. Pe riodismo digital en la Argentina. General Roca: Publifadecs, 2008. http://red-accion.uncoma.edu.ar/PeriodismoDigital/pdf/capitulo8.pdf

Rost, Alejandro. La interactividad en el periódico digital. Tesis doctoral. Barcelona. Univ Autónoma de Barcelona, 2006. http://www.tesisenxarxa. net/TDX-1123106-104448

Rost, Alejandro. "Propuestas para un periódico digital interactivo". Zer, 2007, 22, pp. 269-389.

Rost, Alejandro; Pugni-Reta, María-Emilia. "De la relación de lectores patagónicos con recursos interactivos de periódicos digitales nacionales y regionales". Buenos Aires, 2005. En cd-rom.

Schultz, Tanjev. "Interactive options in online journalism. A content analysis of 100 US newspapers". Journal of computer mediated communication, 1999 , v. 5 , n. 1

http://www.ascusc.org/jcmc/vol5/issue1/schultz.html

Schultz, Tanjev. "Mass media and the concept of interactivity. An exploratory study of online forums and reader email". Media, culture \& society, 2000, v. 22, n. 2, pp. 205-221.

Secko, David M. "The unfinished science story. Reflections on journalistaudience interactions in the online environment". Journal of media practice, 2009, v. 10, n. 2-3, pp. 259-266.

Singer, Jane B. "Contested autonomy". Journalism studies, 2007, v. 8, n. 1, pp. 79-95.

Singer, Jane B. "Quality control". Journalism practice, 2010, v. 4, n. 2, pp. 127-142.

Singer, Jane B.; Ashman, Ian. "Comment is free, but facts are sacred. User-generated content and ethical constructs at The Guardian". Journal of mass media ethics, 2009, n. 24, pp. 3-21.

Spyridou, Paschalia-Lia; Veglis, Andreas. "Exploring structural interactivity in online newspapers. A look at the Greek week landscape". First Monday, 2008, v. 13, n. 5.

http://firstmonday.org/article/view/2164/1960

Stassen, Wilma. "Your news in 140 characters. Exploring the role of social media in journalism". Global media journal African edition, 2010, v. 4, n. 1, pp. $1-16$

http://sun025.sun.ac.za/portal/page/portal/Arts/Departemente1/ Joernalistiek/Global\%20Media\%20Journal/Global\%20Media\%20Journal \%20-\%20Files/8C986D88B97754ABE04400144F47F004

The Bivings Group report. "The use of the internet by America's largest newspapers", 2008. 
http://www.bivingsreport.com/2008/the-use-of-the-internet-by-americaslargest-newspapers-2008-edition/

Thurman, Neil. "From blogrolls and wikis to big business. Going online". En: Keeble, Richard. Print journalism. A critical introduction. London: Routledge, 2005.

Thurman, Neil. "Forums for citizen journalists? Adoption for user generated content initiatives by online news media". New media \& society, 2008, v. 10 , n. 1 , pp. $139-157$.

Thurman, Neil; Hermida, Alfred. "Gotcha. How newsroom norms are shaping participatory journalism online". En: Tunney, Sean; Monaghan, Garrett: Web journalism. A new form of citizenship. Eastbourne. Sussex: Academic Press, 2010.

Tomaiuolo, Nicholas. "U-Content”. Searcher, 2009, 17, n. 9, pp. 12-54.

Vujnovic, Marina; Singer, Jane B.; Paulussen, Steve; Heinonen, Ari; Reich, Zvi; Quandt, Thorsen; Hermida, Alfred; Domingo, David. "Exploring the political-economic factors of participatory journalism. Views of online journalists in 10 countries”. Journalism practice, 2010, v. 4, n. 3, pp. 285-296.

Wunsch-Vincent, Sacha; Vickery, Graham. Participative web. User-created content, working party on the information economy. OECD, Directorate for Science, Technology and Industry, Committee for Information, Computer and Communications Policy, 2007.

http://www.oecd.org/dataoecd/57/14/38393115.pdf

Xiao, Li; Polumbaum, Judy. "News and ideological exegesis in Chinese online media. A case study of crime coverage and reader discussion on two commercial portals". Asian journal of communication, 2006, v. 16, n. 1, pp. $40-58$.

Xu, Xiaoge. "Engaging and empowering news audiences online. A feature analysis of nine Asian news websites". eJournalist, 2009, v. 18, n. 1, pp. 35-53.
Yerzers'ka, Lyudmyla. Ciberperiodismo en Perú, análisis de los diarios digitales. Lima: Editorial San Marcos, 2008.

Yerzers'ka, Lyudmyla. "Tendencias de los diarios digitales en Perú». Revista latina de comunicación social, 2008a, 63, pp. 71-82.

http://www.ull.es/publicaciones/latina/_2008/07/Lyudmyla.html

Zamith, Fernando. Ciberjornalismo. As potencialidades da internet nos sites noticiosos portugueses. Oporto: Ediçoes Afrontamento, 2008.

Zamith, Fernando. "A methodological proposal to analyze the news websites use of the potentialities of the internet". 9th Intl symp on online journalism, 2008a, Univ of Texas, Austin.

http://online.journalism.utexas.edu/2008/papers/Zamith.pdf

Zeng, Qian; Li, Xigen. "Factors influencing interactivity of internet newspapers. A content analysis of 106 US newspapers' web sites”. En: Li, X. (ed.): Internet newspapers. The making of a mainstream medium. Londres: Lawrence Erlbaum Associates, 2006.

Zeta-De-Pozo, Rosa. "Opciones interactivas en el periodismo peruano». Sala de prensa, 2002, n. 50.

http://www.saladeprensa.org

Zúñiga, Danghelly; Duque-Soto, Álvaro-Enrique. "Las herramientas interactivas de dos diarios colombianos". Signo y pensamiento, 2009, v. XXVIII, n. 54, pp. 330-340.

Elvira García-De-Torres, Universidad Cardenal Herrera.

Luis Vives, 1. 46115 Alfara del Patriarca, Valencia. egarcia@uch.ceu.es

elvira72001@yahoo.es

\section{Tu futuro es ahora} Másters y Postgrados IDEC-UNIVERSITAT POMPEU FABRA creemos en ti

\section{Máster en Documentación Digital}

Máster y Programas de postgrado online

Arquitectura de la información en sitios web - Organización y gestión de la información • Buscadores y posicionamiento web - Documentación audiovisual - Web social - Archivística digital - Gestión de contenidos y gestión del conocimiento - Bibliotecas digitales • Usabilidad y accesibilidad.

Dirección: Cristòfol Rovira, Mari Carmen Marcos y Lluís Codina

Calendario: del 4 de octubre de 2010 al 30 de junio de 2011

Programa matriculable por módulos

www.documentaciondigital.org

\section{Máster en Buscadores: Marketing y Posicionamiento}

Máster y Programas de postgrado online

Fundamentos en buscadores - Posicionamiento en Buscadores - SEO - Marketing en Buscadores - SEM

- Analítica web - SEO y SEM en la web social-Vigilancia tecnológica y buscadores especializados.

Dirección: Cristòfol Rovira, Mari Carmen Marcos y Lluis Codina

Calendario: del 11 de noviembre de 2010 al 22 de junio de 2011

Programa matriculable por módulos

www.masterenbuscadores.com

\section{Colaboran:}

Observatorio de Comunicación Científica - UPF Instituto Universitario de Lingüistica Aplicada - UPF DOC 6 Consultores de Recursos de Información
Infórmate:

IDEC-Universitat Pompeu Fabra

www.idec.upf.edu· info@idec.upf.edu

Tel.: +34935421850 • Balmes, 132-134 08008 Barcelona

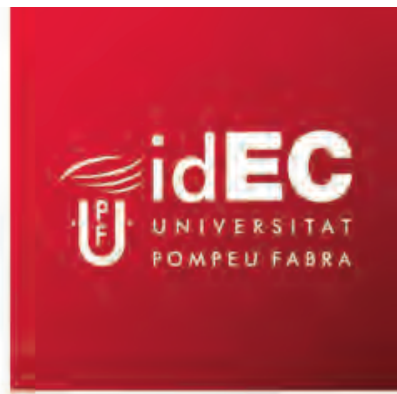

